International gournal of Biological Sciences

2009; 5(5):438-443

Review

(C) Ivyspring International Publisher. All rights reserved

\title{
How Subchronic and Chronic Health Eifects can be Neglected for GVIOs, Pesticides or Chemicals
}

\section{Gilles-Eric Séralini1,2凶, Joël Spiroux de Vendômois², Dominique Cellier ${ }^{2,3}$, Charles Sultan ${ }^{2,4}$, Marcello Buiatti $^{2,5}$, Lou Gallagher 6 , Michael Antoniou 7 , Krishna R. Dronamraju ${ }^{8}$}

1. University of Caen, Institute of Biology, Biochemistry, Esplanade de la Paix 14032 Caen Cedex France.

2. CRIIGEN, 40 rue Monceau, 75008 Paris France

3. University of Rouen, LITIS EA 4108, 76821 Mont Saint-Aignan, France

4. University of Montpellier, School of Medicine, IGH, CNRS, France

5. University of Firenze, Italy

6. Institute for Environmental Science and Research, Ltd, Crown Research Institute, Porirua, New Zealand

7. King's College London School of Medicine, Dept. Medical and Molecular Genetics, London, United Kingdom

8. Foundation for Genetic Research, Houston, USA

Correspondence to: Prof. Gilles-Eric Séralini, PhD, Institute of Biology and CRIIGEN, University of Caen, Esplanade de la Paix, 14032 Caen Cedex, France. Tel +33 2315656 84; Fax +33 25653 20; Email: criigen@unicaen.fr.

Received: 2009.02.26; Accepted: 2009.06.16; Published: 2009.06.17

\begin{abstract}
Chronic health effects are increasing in the world such as cancers, hormonal, reproductive, nervous, or immune diseases, even in young people. During regulatory toxicological subchronic tests to prevent these on mammalian health, prior commercialization of chemicals, including pesticides and drugs, or GMOs, some statistically significant findings may be revealed. This discussion is about the need to investigate the relevant criteria to consider those as biologically significant. The sex differences and the non linear dose or time related effects should be considered in contrast to the claims of a Monsanto-supported expert panel about a GMO, the MON $863 \mathrm{Bt}$ maize, but also for pesticides or drugs, in particular to reveal hormone-dependent diseases and first signs of toxicities.
\end{abstract}

Key words: Pesticides, GMO, MON 863, side effects, toxicological tests.

\section{Introduction}

Some contaminations or pollutions by pesticides [1] and other chemical residues [2-4] affect human and animal health, together with biodiversity. Thus it is important to study potential mid and long-term toxicological effects during regulatory tests prior to commercialization of chemicals, and not to test only short-term or subchronic effects. This question has also been raised for GMOs [5], especially those containing pesticides, either because they tolerate (such as Roundup Ready soya) or produce (such as Bt maize) these molecules ( $99 \%$ of commercially cultivated GMOs). This subject has been reviewed recently by Dronamraju [6].

\section{Objectives}

Here we shall discuss more particularly the existing data on possible toxic effects of a GMO on mammals, with putative relevance to humans, and with the aim of commenting on current procedures and experimental protocols in mammalian feeding experiments (Fig. 1). Doull et al. [7] indicated their general criteria needed to classify as biologically relevant the observed significant effects during 90d toxicological tests on mammals. The example taken was for a GMO, a Bt maize called MON 863, producing in its cells a new kind of modified insecticide Cry3Bb1, known as a toxin for coleopterans. But these 
authors claim to apply the same criteria to other products such as pesticides and drugs. The history of the debate on the biosafety of this GMO is paradigmatic, and it raises a series of general questions on risk assessment of commercial transgenic crops and of pesticides or chemicals. These considerations are cru- cial, since public health is concerned and their discussion may critically influence the decision to release in particular some agricultural GMOs or not, and also to another extent the economic feasibility of this kind of project.

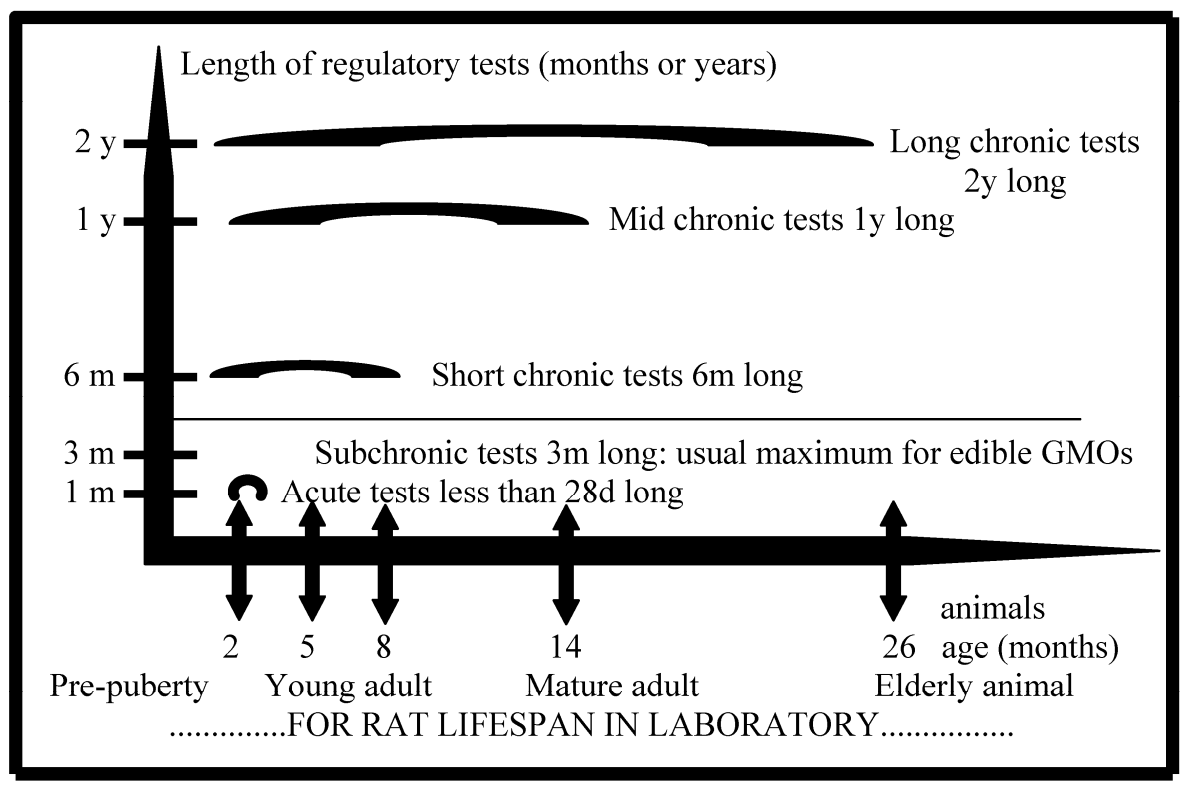

Figure I. Comparison of regulatory toxicity tests generally performed in vivo on mammals, for instance with rats, the most used model, before commercialization of various products. These are GMOs used for food or feed, pesticides, drugs, or the best tested chemicals. The choice of how to apply standards is made by scientific commissions of regulatory instances. This figure does not include reproductive, developmental or trans-generational tests that are not requested for commercialized GMOs for food or feed. Nutritional tests are not represented either because they do not require blood analyses, which are very informative on health secondary effects. Some mammalian nutritional tests are performed with pigs or cows, for instance for GMOs, and may last longer with fewer animals. Subchronic toxicity tests are in the last case performed, if any, only with rats for most GMOs. Then it is only with 10 animals fully assessed on 20 for each of two doses, and per sex. There are 3 mammalian species used for other products. This is to measure short-term effects. The so-called chronic tests (lasting more than 3 months) give more chances to reveal metabolic, nervous, immune, hormonal or cancer diseases. They are widely performed for pesticides and drugs and for some chemicals over a certain production, but not for actual commercialized GMOs released in the environment (1995-2009). This is a matter of debate, since $99.9 \%$ of those are genetically modified to contain new pesticide residues that they tolerate (ex. Roundup Ready soya) or that they produce (ex. insecticides $\mathrm{Bt}$ in maize, that are newly modified proteic toxins). (d: day; m: month; $y$ : year).

\section{The protocol used to test GMOs in regulatory in vivo tests with mammals}

Recently, Doull et al. [7] offered a new contradictory analysis of Séralini et al. [5]. It was about the interpretation of the only crude data available from the longest toxicity test (90 days) on a mammal that had been fed with MON 863. The original feeding experiment was performed by Covance and Monsanto [8], with a great experience of this kind of tests always designed in a similar manner. They measured the effect of feed containing only two doses (11 and $33 \%$ GM in the equilibrated diet) and for only two periods of exposure ( 5 and 14 weeks). The goal is a debate on standards to be set to interpret admitted significant effects [7] between treated groups versus controls as biologically relevant or not in toxicological tests in general.

There are several preliminary unsolved questions at stake to be answered such as whether to prolong tests before commercial release, for instance up to two years for GMOs, as is done for some pesticides or drugs, in order to assess chronic effects not visible in short periods. There are also questions regarding the appropriate number of concentrations of the putative toxic agent to be tested etc., and critical ex- 
perimental criteria such as number of animals to be used per dose or concentration to increase their resolution power to obtain homogeneous and reliable significance levels in outcome measurement data.

However, the crude data on MON 863 were obtained by Monsanto for only one mammalian species (instead of the three used for evaluations of pesticides or drugs) and first classified as confidential by the Company which obtained it (2002). The data was then used to obtain commercial release agreements all over the world. After heated discussions in Europe concerning the possible physiological effects provoked by this GMO, a decision in the German Appeal Court allowed public access to the crude data (2005). Monsanto then published its own interpretation of the data [8] in which it was concluded that the MON 863 was safe to eat.

After careful analysis of the crude data, Séralini et al. [5] applied appropriate statistical methodology to test the effects of the $\mathrm{Bt}$ maize on mammalian health. First, GM fed rats were compared to their closest isogenic controls, and then to the six reference groups who were fed various other maize-based diets that Monsanto added in the study. Data were compiled by organ, dose and timing of dietary exposure. In addition, the effects on the rat metabolism of the diet composition without GM maize was studied, comparing only control and reference groups between them to avoid systematically linking these effects to the GM diet. In the first instance Monsanto did not do such a statistical study ([8] and in commercial request file) but only took into account effects between the GM fed rats at the highest dose and all other groups. It is important to note that in order to isolate the effect of the GM transformation process from other variables it is only valid to compare the GMO (in this case MON 863) with its isogenic non-GM equivalent. Therefore, the inclusion in the analysis of unrelated feeding groups serves to confuse rather than clarify the effect of the MON 863 event.

The goal of the statistical analysis is to decide whether the consumption of GMOs can be considered to have no effect (null hypothesis $\mathrm{H}_{0}$ true) or to have an effect $\left(\mathrm{H}_{0}\right.$ false) on the health of the rats. This analysis cannot be reduced in the computation of a collection of p-values. Statistical rejection of the null hypothesis $\mathrm{H}_{0}$ does not imply that the effect is biologically significant. In the same way, failure to reject $\mathrm{H}_{0}$ does not mean that it is true. Therefore, the power of the hypothesis test must be assessed. The power of a statistical test depends on the sample size (and therefore the experimental design), the significance level of the test and the effect size (which can be considered as biologically significant). This most impor- tant issue is totally overlooked in the experimental design and the statistical report made by Monsanto on MON 863. Moreover, any hypothesis which is not statistically significant with their reductive method is always excluded. This disturbing oversight runs false negative results and a risk of health consequences for millions of people and animals.

\section{Sex-related and non-linear signs of toxicity}

In the MON 863 study, Séralini et al. [5] were also concerned by false positive results, but concluded that there were enough signs of toxicity to prolong the feeding experiments. This is mostly because significant effects were concentrated in livers and kidneys as main detoxification organs reacting in cases of food / chemical contamination; there were at these levels some worrying physiological profiles. Moreover, the effects of the MON 863 insecticide toxin itself are not experimentally documented on mammalian cells. Furthermore, it remains a possibility that there would be side effects due to insertional mutagenesis during the GM transformation. For instance, the Séralini et al. [5] analysis showed evidence of a significant increase in blood glucose of $10 \%$ in GM-fed females, in triglycerides of $24-40 \%$, overweight livers and enhanced liver/brain ratios $(7 \%)$, small but significant body weight gain $(3.7 \%)$, and disturbed kidney parameters. When comparing females eating GMOs to their closest controls eating the isogenic line, there were signs of a possible pre-diabetic profile. In both sexes and periods the profiles were different but it concerned liver and kidney parameters.

From that, Doull et al. [6] concluded that any effects with no clear dose-response relationship (which should increase with dose) or with time are unrelated to the GM diet. We consider that first of all, to a scientific point of view, choosing a priori 2 doses and 2 periods does not allow the assessment of a linear dose- or period-related effect [9-11]. Our hypothesis was to question the possibility of subchronic or chronic health effects that were not or only partially revealed by short-term tests. Several hormonal disrupting effects do not linearly increase with time or dose, but present non-linear peaks in the shape of $U$ or J curves [12-14] at some periods or some ranges of doses, depending on the age and exposure period of the test animals [15-17]. Secondly, a clear histopathological study should be published and studied in parallel to the biochemical effects found by Monsanto or the Monsanto-supported expert panel [7]. It is possible that metabolic changes precede, within 90 days, histopathological lesions that could appear afterwards. This is another reason to prolong the experiments and may also solve the problem of reproduci- 
bility. Simultaneously, the occurrence of similar effects in both sexes is an important criterion of toxicity for Doull et al. [7], which is not for us. Sex-dependent differences in chronic diseases resulting from chemical intoxication are well established $[18,19]$. The liver is itself a sex-differentiated organ; for example chemical sensitivity is different in males and females [20]. In Monsanto's data for these 3 month rat tests with Bt maize MON 863, for all rats including the six times bigger group of normal control and reference animals eating non GM diets, there were confirmed sex-differentiated effects in liver and kidneys parameters (Fig.2A and B). Doull et al. [7] also considered a normal range of variations in undefined historical data, or compiled the closest isogenic control with other reference groups that have diets different in salt or sugars. This is not scientifically precise.
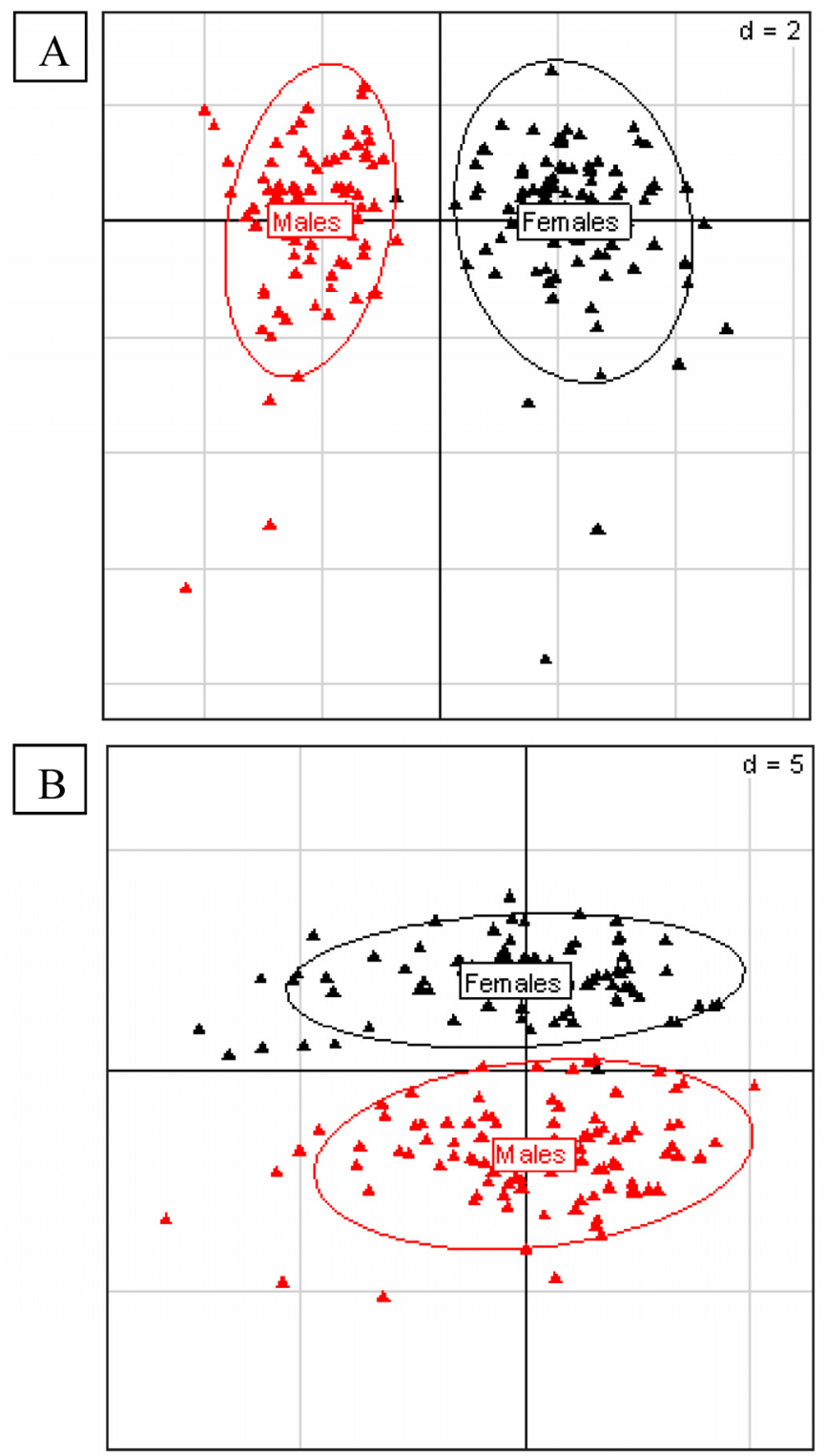

Figure 2. A. Principal Component Analysis for liver parameters in all rats of the MON 863 experiment. It was performed according to Hotelling [29] in order to study the scattering of the different factors. The scheme obtained for parameters at week I 4 explains $42.42 \%$ of the total data variability (inertia) expressed on 2 axes ( $32.01 \%$ for factor I; $10.41 \%$ for factor 2 ), scale $d=2$. This demonstrates the clear separation of parameters values according to sex. B. Principal Component Analysis for kidney parameters in all rats of the MON 863 experiment. The scheme obtained for parameters at week 14 explains $47.73 \%$ of the total data variability (inertia) expressed on 2 axes $(26.95 \%$ for factor I; $20.78 \%$ for factor 2 ), scale $d=5$. This demonstrates the clear separation of parameters values according to sex. 


\section{Conclusion}

We assume that Séralini et al. [5] methodology can discriminate potential false positive and GM-linked effects, avoiding to some extent false negative ones, in the best way we can do for this discussed and too limited protocol already in use for commercialized GMOs. These GM-linked effects are then considered as signs of toxicity in the 90 days, not proofs of toxicity. The biological plausibility of a subchronic or chronic side effect of the GM diet, linked to the new toxin in the mammalian regimen, or due to the mutagenesis effect of the genetic modification itself, is thus non negligible. Finally it should be stressed that statistically significant effects of GM diets, or of residues of pesticides that are contained by GMOs, have also been observed in other instances [21-25], but not in all studies [26, 27] enlightening the necessity of a case-by-case approach, and that the real toxicological studies are quite limited up to date for that [28]. All these observations taken together in our opinions do not allow a clear statement of toxic effects, but to suggest them as such, because they are clearly undeniable. Now, to any good researcher similar results would mean that there is much to be improved in the planning of experimental design; and thus to increase their resolution power to obtain unequivocal statements, for instance increasing the duration and/or the number of rats tested. Generally speaking it seems to us unbelievable that a risk assessment carried out only on forty rats of each sex receiving GM rich diets for 90 days (yielding results often at the limits of significance) have not been repeated and prolonged independently. We should overall take into account the fact that the analysed GM product could be fed long-term to people and animals of various ages and sexes, and with various pathologies.

We call for more serious standardized tests such as those used for pesticides or drugs, on at least three mammalian species tested for at least three months employing larger sample sizes, and up to one and two years before commercialization, for GM food or feed specifically modified to contain pesticide residues. We also call for a serious scientific debate about the criteria for testing significant adverse health effects for pesticides or chemicals, but overall for GM food or feed products, such as MON 863.

\section{Acknowledgements}

We thank François Roullier for Fig. 2 and statistical studies.

\section{Conflict of Interest}

The authors have declared that no conflict of interest exists.

\section{References}

1. Daston GP, Cook JC, Kavlock RJ. Uncertainties for endocrine disrupters: our view on progress. Toxicol Sci. 2003; 74: 245-52.

2. Toppari J, Larsen JC, Christiansen $P$, et al. Male reproductive health and environmental xenoestrogens. Environ Health Perspect. 1996; 104: 741-803.

3. Paris F, Jeandel C, Servant $\mathrm{N}$, et al. Increased serum estrogenic bioactivity in three male newborns with ambiguous genitalia: a potential consequence of prenatal exposure to environmental endocrine disruptors. Environ Res. 2006; 100: 39-43.

4. Belpomme D, Irigaray P, Hardell L, et al. The multitude and diversity of environmental carcinogens. Environ Res. 2007; 105: 414-29.

5. Seralini GE, Cellier D, Spiroux de Vendomois J. New analysis of a rat feeding study with a genetically modified maize reveals signs of hepatorenal toxicity. Arch Environ Contam Toxicol. 2007; 52: 596-602.

6. Dronamraju K.R. Emerging Consequences of Biotechnology. New Jersey, USA: World Scientific Publishing Co. 2008.

7. Doull J, Gaylor D, Greim HA, et al. Report of an expert panel on the reanalysis by Séralini et al. (2007) of a 90-day study conducted by Monsanto in support of the safety of a genetically modified corn variety (MON 863). Food Chem Toxicol. 2007; 45: 2073-2085.

8. Hammond B, Lemen J, Dudek R, et al. Results of a 90-day safety assurance study with rats fed grain from corn rootworm-protected corn. Food Chem Toxicol. 2006; 44: 147-160.

9. Green S. Toxicology and regulatory process. New York, USA: Taylor and Francis Group; 2006.

10. Nielsen E, Østergaard G, Larsen JC. Toxicological Risk Assessment of Chemicals - A Practical Guide. New York, USA: Taylor and Francis Group; 2008.

11. Gaylor D, Chen J, Kodell R. Experimental Bioassays for Screening and Low Dose Extrapolation. Risk Analysis 1985; 5: 9-16.

12. Andersen $\mathrm{ME}$, Barton HA. Biological regulation of receptor-hormone complex concentrations in relation to dose-response assessments for endocrine-active compounds. Toxicol Sci.1999; 48: 38-50.

13. Bencko V. Human exposure to endocrine disrupters: carcinogenic risk assessment. Folia Histochem Cytobiol. 2001; 39 (Suppl 2): 24-5.

14. Melnick R, Lucier G, Wolfe M, et al. Summary of the National Toxicology Program's report of the endocrine disruptors low-dose peer review. Environ Health Perspect. 2002; 110: 427-31.

15. Karanth S, Pope C. Carboxylesterase and A-esterase activities during maturation and aging: relationship to the toxicity of chlorpyrifos and parathion in rats. Toxicol Sci. 2000; 58: 282-9.

16. Howard MD, Mirajkar N, Karanth S, et al. Comparative effects of oral chlorpyrifos exposure on cholinesterase activity and muscarinic receptor binding in neonatal and adult rat heart. Toxicology. 2007; 238: 157-65.

17. Benbrahim-Tallaa L, Siddeek B, Bozec A, et al. Alterations of Sertoli cell activity in the long-term testicular germ cell death process induced by fetal androgen disruption. J Endocrinol. 2008; 196: 21-31. 
18. Lu SC, Kuhlenkamp J, Garcia-Ruiz C, et al. Hormone-mediated down-regulation of hepatic glutathione synthesis in the rat. J Clin Invest. 1991; 88: 206-269.

19. Sissung TM, Price DK, Sparreboom A, et al. Pharmacogenetics and regulation of human cytochrome P450 1B1: Implications in hormone-mediated tumor metabolisme and a novel tagert for therapeutic intervention. Mol Cancer Res. 2006; 4: 135-150.

20. Kobliakov V, Popova N, Rossi L. Regulation of the expression of the sex-specific isoforms of cytochrome P-450 in rat liver. Eur J Biochem. 1991; 195: 585-91.

21. Malatesta M, Caporaloni C, Gavaudon S, et al. Ultrastructural morphometrical and immunocytochemical analyses of hepatocyte nuclei from mice fed on genetically modified soybean. Cell Struct Function. 2002; 27: 173-180.

22. Malatesta M, Biggiogera $M$, Manuali E, et al. Fine structural analyses of pancreatic acinar cell nuclei from mice fed on genetically modified soybean. Eur J Histochem. 2003; 47: 385-388.

23. Vecchio L, Cisterna B, Malatesta M, et al. Ultrastructural analysis of testes from mice fed on genetically modified soybean. Eur J Histochem. 2004; 48: 449-454.

24. Trabalza-Marinucci M, Brandi G, Rondini C, et al. A three-year longitudinal study on the effects of a diet containing genetically modified Bt176 maize on the health status and performance of sheep. Livestock Sci. 2008; 113: 178-190.

25. Benachour N, Sipahutar H, Moslemi S, et al. Time- and dose-dependent effects of roundup on human embryonic and placental cells. Arch Environ Contam Toxicol. 2007; 53: 126-133.

26. Brake DG, Evenson DP. A generational study of glyphosate-tolerant soybeans on mouse fetal, postnatal, pubertal and adult testicular development. Food Chem Toxicol. 2004; 42: 29-36.

27. Brake DG, Thaler R, Evenson DP. Evaluation of Bt (Bacillus thuringiensis) corn on mouse testicular development by dual parameter flow cytometry. J Agric Food Chem. 2004; 52: 2097-2102.

28. Domingo JL. Toxicity studies of genetically modified plants: A review of the published literature. Crit Rev Food Sci Nut. 2007; 47: 721-733.

29. Hotelling H. Analysis of a complex of statistical variables into principal components. J Educ Psychol. 1933; 24: 417-441. 